\title{
Lithium abundances along the red giant branch: FLAMES-GIRAFFE spectra of a large sample of low-mass bulge stars $\star, \star \star$
}

\author{
T. Lebzelter ${ }^{1}$, S. Uttenthaler ${ }^{1, \star \star \star}$, M. Busso ${ }^{2}$, M. Schultheis ${ }^{3}$, and B. Aringer ${ }^{4}$ \\ 1 University of Vienna, Department of Astronomy, Türkenschanzstraße 17, 1180 Vienna, Austria \\ e-mail: [thomas.lebzelter; stefan.uttenthaler]@univie.ac.at \\ 2 Dipartimento di Fisica, Università di Perugia, and INFN, Sezione di Perugia, via Pascoli, 06123 Perugia, Italy \\ 3 Observatoire de Besançon, 41bis, avenue de l'Observatoire, 25000 Besançon, France \\ 4 INAF - Padova Astronomical Observatory, Vicolo dell'Osservatorio 5, 35122 Padova, Italy
}

Received 21 July 2011 / Accepted 7 November 2011

\begin{abstract}
Context. A small number of K-type giants on the red giant branch (RGB) is known to be very rich in lithium (Li). This fact is not accounted for by standard stellar evolution theory. The exact phase and mechanism of Li enrichment is still a matter of debate. Aims. Our goal is to probe the abundance of $\mathrm{Li}$ along the RGB, from its base to the tip, to confine Li-rich phases that are supposed to occur on the RGB.

Methods. For this end, we obtained medium-resolution spectra with the FLAMES spectrograph at the VLT in GIRAFFE mode for a large sample of 401 low-mass RGB stars located in the Galactic bulge. The Li abundance was measured in the stars with a detectable Li $670.8 \mathrm{~nm}$ line by means of spectral synthesis with COMARCS model atmospheres. A new $2 \mathrm{MASS}\left(J-K_{\mathrm{S}}\right)-T_{\text {eff }}$ calibration from COMARCS models is presented in the Appendix.

Results. Thirty-one stars with a detectable Li line were identified, three of which are Li-rich according to the usual criterion $(\log \epsilon(\mathrm{Li})>1.5)$. The stars are distributed all along the RGB, not concentrated in any particular phase of the red giant evolution (e.g. the luminosity bump or the red clump). The three Li-rich stars are clearly brighter than the luminosity bump and red clump, and do not show any signs of enhanced mass loss.

Conclusions. We conclude that the Li enrichment mechanism cannot be restricted to a clearly defined phase of the RGB evolution of low-mass stars $\left(M \sim 1 M_{\odot}\right)$, contrary to earlier suggestions from disk field stars.
\end{abstract}

Key words. stars: late-type - stars: evolution - stars: abundances

\section{Introduction}

Lithium (Li) is an important diagnostic tool in stellar evolution because its abundance strongly depends on the ambient conditions. It is quickly destroyed at $T>3 \times 10^{6} \mathrm{~K}$ so that it diminishes if the stellar surface is brought into contact with hot layers by mixing processes. However, if the overturn time scale for mixing becomes faster than the decay of the parent ${ }^{7} \mathrm{Be}$, then Li destruction reverts into production through the socalled Cameron-Fowler mechanism (Cameron \& Fowler 1971). In low-mass stars during the main-sequence phase, the initial Li abundance strongly decreases, hence slow mixing should prevail (Michaud 1986). During the ascent on the red giant branch (RGB), any Li remaining in the envelope is further diluted by the first dredge-up (FDU); stellar models excluding atomic diffusion and rotation predict a surface $\mathrm{Li}$ abundance at FDU of $\log \epsilon(\mathrm{Li}) \leq+1.5($ where $\log \epsilon(\mathrm{Li})=\log [N(\mathrm{Li}) / N(\mathrm{H})]+12)$ at this stage. This is indeed what is observed in most G-K giants (Lambert et al. 1980; Brown et al. 1989; Mishenina et al. 2006),

* Based on observations at the Very Large Telescope of the European Southern Observatory, Cerro Paranal/Chile under Programme 083.D-0046(A).

$\star \star$ Table 1 is only available at the CDS via anonymous ftp to cdsarc.u-strasbg.fr (130.79.128.5) or via

http://cdsarc.u-strasbg.fr/viz-bin/qcat?]/A+A/538/A36

$\star \star \star$ The first two authors have contributed equally to this paper. where some of these stars show Li even far below the expectations (Mallik 1999). However, about 1-2\% of the $\mathrm{K}$ giants have a high Li abundance and are therefore called Li-rich (Brown et al. 1989; de La Reza et al. 1997).

Advanced evolutionary stages in which Li abundances might possibly increase through quite fast mixing have been identified by Charbonnel \& Balachandran (2000, hereafter CB00), in the moments at which the discontinuity of molecular weight $\mu$ left behind by the downward envelope expansion is erased by the advancement of the H-burning shell. This occurs at the bump of the luminosity function, which is on the RGB for low masses. For intermediate mass stars, this moment is postponed until after core He-burning, when the star climbs for the second time towards the Hayashi track and the envelope deepens again (on the so-called asymptotic giant branch, or AGB). The absence of a $\mu$-barrier is the key factor because it allows any diffusive or plume-like mixing mechanism to link the envelope with inner layers, and in some cases does it fast enough, to activate a Cameron-Fowler mechanism for Li production. However, the proposed location of the enrichment phase directly at the RGB bump has been questioned by recent observational results (e.g. Monaco et al. 2011). Observations of $\mathrm{Li}$ in metal-poor globular cluster stars show that a slow extra-mixing episode at the RGB bump efficiently destroys any Li remaining after FDU (Lind et al. 2009b). Hence, evolved Li-rich stars brighter than the RGB bump are evidence that those stars somehow skip this mixing episode or even 
undergo a phase of Li production, which motivates the search for these stars. As it is unlikely that they skipped Li destruction at FDU, they must have somehow replenished it through Be decay (see the discussion in Palmerini et al. 2011).

While the Li abundance has been measured already in an increasing number of field stars (e.g. Mallik 1999; Kumar et al. 2011; Monaco et al. 2011) the samples investigated always had the disadvantage of either inhomogeneity, especially in mass and age, uncertainty of the distance, or strong selection biases (e.g. only stars around the RGB bump). This circumstance hampers drawing definite conclusions on the evolutionary phase and length of the $\mathrm{Li}$ enrichment. Homogeneous samples, such as RGB stars in a cluster, are small (e.g. Pasquini et al. 2001, 2004). We therefore designed a programme to spectroscopically observe a large and homogeneous sample of stars from the bottom to the tip of the RGB belonging to the Galactic bulge (GB) to derive their $\mathrm{Li}$ abundances. The GB offers a large number of low-mass giants at roughly equal distance within a small area in the sky, perfectly suited for observations with a multi-object spectrograph such as FLAMES-GIRAFFE. Furthermore, in contrast to globular clusters, the Bulge contains stars in a wide range of metallicities, which provides the opportunity to check for any dependence of the $\mathrm{Li}$ enrichment processes on this parameter. We present the results of this programme concerning our search for Li. It will not only help to confine the phases and duration of $\mathrm{Li}$ production on the $\mathrm{RGB}$, but will also provide a reference value for the $\mathrm{Li}$ abundance in the outer $\mathrm{GB}$, which is needed for interpreting the first Li-rich AGB stars detected there recently (Uttenthaler et al. 2007, hereafter U07). The present data set is also of high interest for the study of the structure and nature of the bulge itself, the results of which will be presented in a forthcoming paper (Uttenthaler et al., in prep.).

\section{Sample and observations}

\subsection{Target selection}

The selection of targets was based on data from the 2MASS catalogue (Skrutskie et al. 2006) in a 25' diameter circle towards the direction $(l, b)=\left(0^{\circ},-10^{\circ}\right)$, which is the centre of the PalomarGroningen field no. 3 (PG3). This field in the outer GB was chosen because the sample of AGB stars studied by U07 is also located in the PG3. The GB stars in this field are roughly $1.4 \mathrm{kpc}$ away from the Galactic plane and centre. A colour-magnitude diagram (CMD) to illustrate the target selection is displayed in Fig. 1. The observed targets (black circles) were chosen to fall close to two isochrones from Girardi et al. (2000), which are representative for the GB: $Z=0.004$ and age $10 \times 10^{9}$ years, and $Z=0.019$ (which is $Z_{\odot}$ on the scale used by Girardi et al. 2000 ) and age $5 \times 10^{9}$ years. A distance modulus of 14.5 to the GB was adopted. The chosen targets were allowed to have a $(J-K)_{0}$ colour either bracketed by the isochrones, or $0 \mathrm{~m} 02$ redder or bluer than these. We dereddened the stars using the linear relation for the reddening in the $B_{J}$ photographic band as a function of galactic latitude given in Schultheis et al. (1998). To translate this into the extinction in the $J$ - and $K$-bands, we used the relation $R=A_{V} / E(B-V)$ with $R=3.2$ and the reddening law of Glass \& Schultheis (2003). We find a mean reddening of 0.129 in the $J$-band and 0.049 in the $K$-band. A slightly higher reddening is found using the map of Schlegel et al. (1998), but the difference is within the errors of the 2MASS photometry. In addition to the selection with the colour criterion we selected only stars fainter than $J_{0}=9$. 0 to exclude AGB stars above the tip of the RGB, and stars brighter than $J_{0}=14$. 5 to include the RGB

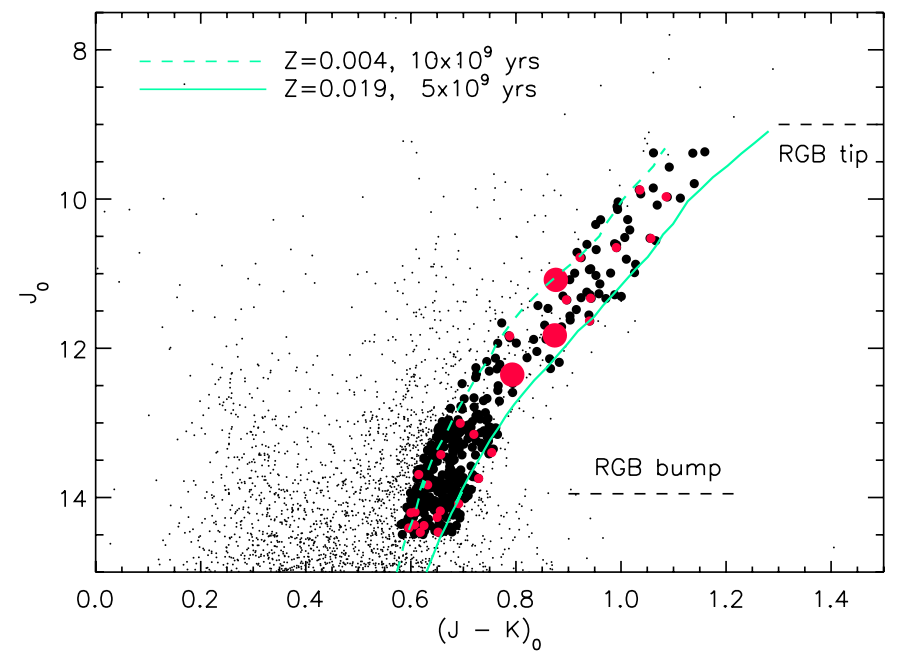

Fig. 1. 2MASS Colour-magnitude diagram of the field around $(l, b)=$ $\left(0^{\circ},-10^{\circ}\right)$. Stars not observed in the present programme are plotted as black dots, observed targets as black circles, stars moderately enriched in Li as small red circles, and Li-rich stars as big red circles. Two RGB isochrones from Girardi et al. (2000), with metallicities and ages as indicated in the legend, are also included. The isochrones are truncated at the tip of the RGB.

bump, which is expected from isochrones at $13.8 \leqslant J_{0} \leqslant 14^{\mathrm{m}}$. 1 . Furthermore, we discarded all targets that had fewer than two quality flags " $\mathrm{A}$ " in the 2MASS JHK photometry and targets that had another source within $3^{\prime \prime}$ that was not fainter by at least 2 . 0 in the $J$-band than the target itself. This selection scheme provides a bias against close visual pairs in our sample. Applying all those criteria yielded 514 targets for the observations.

Also discernible in Fig. 1 are the two red clumps (RCs) recently identified by Nataf et al. (2010) and McWilliam \& Zoccali (2010), which probably represent two populations at different distance in the GB, which are of similar age and metallicity (De Propris et al. 2011). These structures were not known to us in the design phase of the programme. Initially, the fainter RC at $J_{0} \sim 13$. 9 was interpreted by us to be the RGB bump, and only the brighter one at $J_{0} \sim 13$. 2 as the red clump. This recent discovery introduces an uncertainty of a bulge star's location on the RGB of about 0.7 . The locations of the RGB bumps of the two populations are then expected to be slightly above and below the theoretical line marked in Fig. 1. Given this range of distance moduli ( $\sim 14^{\mathrm{m}} .1$ to $\sim 14^{\mathrm{m}} .8$ ) and the photometric errors from the 2MASS catalogue, we can estimate the expected range of metallicities in our sample with the help of the isochrones. We find that at the lower brightness end, stars down to a metallicity of $[\mathrm{M} / \mathrm{H}]=-1.3$ would still fall in the selection range, albeit with a lower probability than stars with a metallicity between that of the two selection isochrones. At brighter magnitudes, the lower metallicity cut will be somewhat higher, but stars down to $[\mathrm{M} / \mathrm{H}]=-0.7$ should be complete in the whole magnitude range of our sample. On the other hand, stars belonging to the near side of the X-shaped bulge may still fall within our selection region up to a metallicity of $[\mathrm{M} / \mathrm{H}]=+0.2$. As we will see in Sect. 3, a few stars fall outside this range, but those cases should be rare.

\subsection{Observations and data reduction}

To measure the Li abundance we obtained spectra of the sample stars using the FLAMES spectrograph in the GIRAFFE configuration. The grating HR15 centred at $665 \mathrm{~nm}$ was used, 
which gave a spectral resolution of 17000 for a wavelength coverage from 644 to $682 \mathrm{~nm}$. This setting contains the Li resonance line at $670.8 \mathrm{~nm}$ and the $\mathrm{H} \alpha$ line, in addition to several other atomic and molecular features. Because our sample stretches over a brightness range of 5 magnitudes, it was divided into three sub-groups, a bright (11. $9 \lessgtr R \lesssim 13$. 3$)$, an intermediate $\left(13.3 \lessgtr R \lessgtr 14^{\mathrm{m}} \cdot 7\right)$, and a faint group $\left(14^{\mathrm{m}} \cdot 7 \lessgtr R \lesssim 16^{\mathrm{m}} 2\right)$, with total exposure times of $0.5,1$, and $4 \mathrm{~h}$, respectively ${ }^{1}$. For the bright group one fibre configuration was sufficient, while the larger number of targets at fainter magnitudes made necessary to split up the intermediate and faint group into two and three fibre configuration, respectively. The observations were obtained in service mode in June 2009. To keep observing blocks below one hour and to handle cosmic ray hits, the observations of the intermediate group were split into two, those of the faint group into five exposures. Individual spectra were extracted from each of these images separately using the FLAMES pipeline provided by ESO. The quality of each spectrum was checked, and those with low signal or cosmic ray hits in the critical wavelength ranges were rejected. Before averaging the good spectra, small wavelength shifts caused by splitting the individual observations in some cases over a few weeks were corrected. The applied shift agreed with the expected value for the difference in heliocentric correction, and the same shift was found for all spectra obtained at a given time. Therefore, we can exclude that these shifts are the result of orbital motion in a binary system. No other velocity shifts were found within the accuracy limits of our data. However, for several stars only one observation was available, therefore we point out that with the present data set an effective discrimination against binaries is not possible. We aimed at a signal-to-noise ratio $(\mathrm{S} / \mathrm{N})$ close to 100 , which was achieved for most targets. Of the initially proposed sample, 401 spectra had sufficiently high quality to check for the presence of the Li line and to measure their radial velocity (RV).

\subsection{Foreground contamination}

An important point for this kind of study is the foreground contamination of the sample. To reliably estimate the fraction of genuine bulge stars in our sample, we ran two simulations of Galactic population models, namely the Besançon model (Robin et al. 2003) and the TRILEGAL model (Girardi et al. 2005). For the TRILEGAL simulation (kindly provided by Vanhollebeke), the best-fit parameters of a tri-axial bulge found by Vanhollebeke et al. (2009) were adopted. We applied the same colour and magnitude selection criteria to the simulated data as to the observed data. This selection yielded $81 \%$ and $74 \%$ bulge stars in the TRILEGAL and Besançon model, respectively. We also inspected the distribution of initial masses of the simulated stars in the selection area. The mass spectrum in the TRILEGAL simulation is relatively sharply peaked at $1.01 M_{\odot}$, with a standard deviation of $0.13 M_{\odot}$. Hence, we are confident that our sample indeed contains mostly low-mass $\left(M \sim 1 M_{\odot}\right)$ red giant stars that are located in the GB.

Some of the foreground stars might be identified by their high proper motion. We therefore searched the Southern Proper Motion Program catalogue version 4 (SPM4; Girard et al. 2011) for sample stars with high proper motion. All but ten of our sample stars were identified in the SPM4 catalogue. Unfortunately, the uncertainties in the SPM4 catalogue are large, so that bulge and foreground stars can hardly be separated. We decided to as-

\footnotetext{
1 The $R$-band limits were determined from the isochrone predictions and used in the GIRAFFE exposure time calculator.
}

sign those stars to the foreground whose proper motion is higher than 20 mas/yr. Applying this criterion, we found 50 foreground star candidates; these stars are marked with an asterisk in Table 1. One star in our sample (\#300) has a particularly high proper motion of more than $200 \mathrm{mas} / \mathrm{yr}$, hence it is probably a nearby $\mathrm{K}$ dwarf.

\section{Analysis and results}

For the following analysis we used hydrostatic COMARCS atmospheres in connection with the COMA spectral synthesis package (Aringer et al. 2009). This ensures a consistent treatment of radiative transfer and opacities during the complete analysis. We started with a temperature calibration based on 2MASS $\left(J-K_{\mathrm{S}}\right)_{0}$ colours, which is presented in Appendix A. A determination of the stellar temperature and surface gravity using only the FLAMES spectra was not possible because of the limited spectral range and the dominance of $\mathrm{TiO}$ lines in the cooler targets. After fixing the temperature based on the colours, we obtained the surface gravities $(g)$ from isochrones in Fig. 1 (Girardi et al. 2000), assuming that the stars belong to the Bulge RGB. The uncertainty in the distance modulus of \pm 0 m.35 (Sect. 2) introduces an uncertainty in $\log (g)$ of \pm 0.16 , which has only a very minor impact on the uncertainty in the $\mathrm{Li}$ abundance, however.

In a first step of the analysis of the spectra, we determined the RVs of the stars by cross-correlating the observed spectra with a synthetic spectrum that was based on a COMARCS model with a temperature close to that estimated for each star. In the next step, the observed spectra were inspected visually around the $670.8 \mathrm{~nm}$ Li line, together with a synthetic spectrum calculated with no Li to check for excess absorption. The Li line was found to be detectable in 31 stars. Four of these Li-bearing stars are also foreground star candidates (Sect. 2).

To estimate the metallicity of each star, we calculated model atmospheres with five different metallicities $([\mathrm{M} / \mathrm{H}]=-1.5$, $-1.0,-0.5,0.0$, and +0.5$)$, with $T_{\text {eff }}$ and $\log g$ values determined in the previous step. A general $\alpha$-element enhancement of $[\alpha / \mathrm{Fe}]=+0.2$, a $\mathrm{C} / \mathrm{O}$ ratio of 0.3 , and a micro-turbulent velocity typical for red giant stars of $\xi=2.5 \mathrm{~km} \mathrm{~s}^{-1}$ were assumed. The spectra with the five different metallicities were interpolated and fitted to the observed spectra in the wavelength range $649-680 \mathrm{~nm}$ using the downhill simplex IDL routine amoeba.pro. The aim of this exercise was not to determine a very precise metallicity of the stars, but instead to reasonably model the quasi-continuum in the vicinity of the Li line, which is suppressed from the true continuum by a forest of weak lines, and the lines blending with the $\mathrm{Li}$ line. This procedure resulted in very satisfying fits of the spectra. The metallicity determined in this way is probably quite reliable, its accuracy is mainly limited by the accuracy of the temperature determination. A check of this method on the observed spectrum of Arcturus yields a metallicity of $[\mathrm{M} / \mathrm{H}]=-0.69$. However, if model atmospheres without an alpha-enhancement are used, this would be increased to -0.64 , which reasonably agrees with the range of metallicities found for this star in the Simbad database. We find a mean metallicity of $[\mathrm{M} / \mathrm{H}]=-0.48$ for the $31 \mathrm{Li}$-bearing stars, which is somewhat lower than what would be expected from previous investigations of Bulge fields close to ours (Zoccali et al. 2008) ${ }^{2}$. Some of the stars have very weak metal lines, indeed. Possibly, there are selection effects introduced in the sense that the Li-rich phenomenon could be more common among metal-poor stars,

2 Performing the averaging on a linear scale, which is the mathematically correct way, the mean is $[\mathrm{M} / \mathrm{H}]=-0.21$. 
and/or that the detection threshold of $\mathrm{Li}$ is lower at lower metallicity because of the reduced background of blending lines. Note that the three most Li-rich stars (see below) have a metallicity between -0.83 and -0.96 dex below solar.

With the stellar parameters fixed in this way, we synthesised spectra, assuming varying $\mathrm{Li}$ abundances to fit the observed spectra. Atomic line data for the spectral synthesis were taken from the VALD data base (Kupka et al. 1999). The line list was checked by comparing a model spectrum of Arcturus (adopting the stellar parameters and abundances found by Ryde et al. 2010) with the observed Arcturus spectrum (Hinkle et al. 2000) in the vicinity of the $671 \mathrm{~nm} \mathrm{Li}$ line. Some lines present in VALD were found to be too strong in the model spectrum; their $\log (g f)$ were decreased accordingly, or the line was completely removed from the list. The abundances given in Caffau et al. (2009) were adopted as solar reference. We did not apply NLTE corrections to the Li abundances (Lind et al. 2009a) derived under LTE assumption because these corrections are usually small and, for the parameters covered by our sample stars, within the uncertainties.

The programme stars \#042, \#080, and \#123 are identified to be Li-rich according to the classical definition, one of them (\#042) has a Li abundance that agrees with the cosmic value and should be regarded as super Li-rich. The observed spectrum of star \#042 around the Li line together with a synthetic spectrum with the best fitting abundances is displayed in Fig. 2. The spectrum of a Li-detected star $(\# 030, \log \epsilon(\mathrm{Li})=+0.1)$ and of a Li-poor star (\#051) are also shown in that figure. All other stars with a detected Li line have an abundance at or below the solar abundance. The error in the Li abundance was estimated by varying the stellar parameters by their uncertainty. The temperature has the highest influence on the strength of the Li line, and is uncertain by $\pm 160 \mathrm{~K}$ for the hotter stars, and $\pm 90 \mathrm{~K}$ for the cooler stars, based on the uncertainty in the 2MASS photometry. We consequently estimate an uncertainty in the $\mathrm{Li}$ abundance of \pm 0.3 dex. Moreover, the detection threshold varies with the temperature because Li becomes more and more ionised at higher temperature. A detection threshold of $\log \epsilon(\mathrm{Li})=+0.5$ was derived for the hottest sample stars $(4800 \mathrm{~K})$, which declines to 0.0 at around $4200 \mathrm{~K}$, and to $\sim-0.5$ at $3600 \mathrm{~K}^{3}$.

All measured $\mathrm{Li}$ abundances as well as important quantities of the targets are collected in Table 1. Our results are also illustrated in Fig. 1: Stars with a detected Li line are plotted as small red circles, and the Li-rich stars are plotted as large red circles.

\section{Discussion}

\subsection{The frequency of Li rich stars}

The three most Li-rich stars in our sample are located on the upper RGB, clearly above the bump and the two RCs. The faintest of our Li-rich stars (\#123), at a $K$-magnitude of 11 m. 61 , is more than one magnitude brighter than the bright $\mathrm{RC}$ (peak at $K \sim 12$ m.65). Taking into account the $K$-magnitude spread of the RCs of 0.22 (David Nataf, priv. comm.), this means that the faintest Li-rich star is brighter than the bright RC peak by 4.7 standard deviations. Hence, even considering the error in distance caused by the spread within the Bulge, it is extremely unlikely that the Li-rich stars are related to either the RGB bump or clump. On the other hand, the Li-detected stars are scattered all along the RGB, from below the bump to the tip. Neither at the bump nor at the RCs do we see an overdensity of Li-detected

\footnotetext{
3 At still lower temperatures, the detection threshold rises again because of the increasing strength of the TiO lines.
}

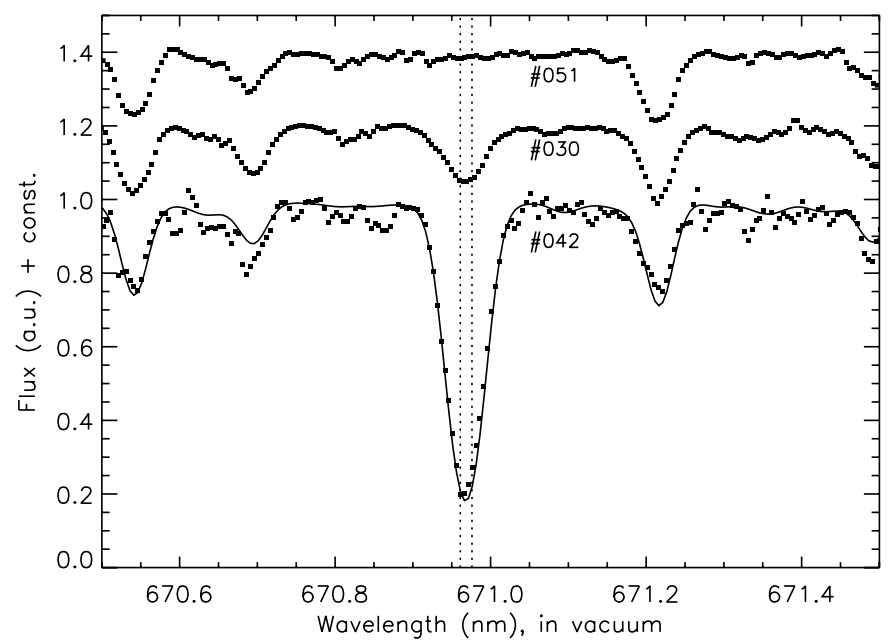

Fig. 2. Observed spectra of the the programme stars \#042, the most Lirich star in our sample; \#030, a Li-detected star; and \#051, a Li-poor star (black dots, from bottom to top). For clarity, the spectra of stars $\# 030$ and \#051 have been shifted by +0.2 and +0.4 in flux. The continuous line is the best-fit synthetic spectrum to star \#042 with a Li abundance of $\log \epsilon(\mathrm{Li})=+3.2$. The two dotted vertical lines indicate the laboratory wavelengths of the hyperfine transitions in ${ }^{7} \mathrm{Li}$. Note also the good fit to two Fe lines at $\sim 670.55$ and $\sim 671.21 \mathrm{~nm}$, which indicates a well-determined metallicity of $[\mathrm{M} / \mathrm{H}]=-0.85$ for star $\# 042$. The metallicities for the two other stars were determined to be -0.94 (\#030) and -1.03 (\#051), respectively.

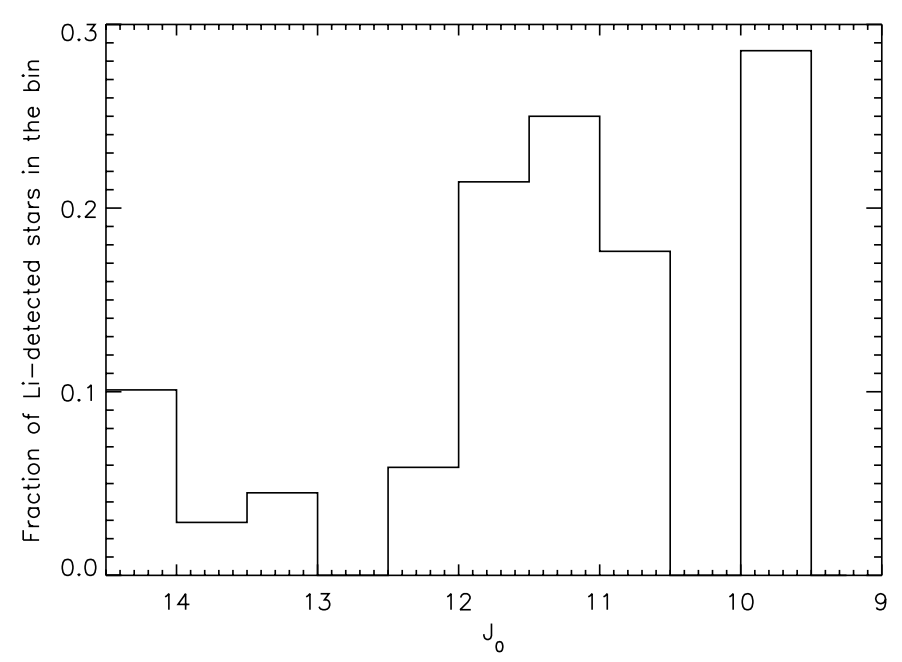

Fig. 3. Fraction of Li-detected stars as a function of $J_{0}$.

stars. In contrast, the fraction of Li-detected stars on the upper RGB $\left(J_{0}<12\right.$ m 0$)$ is $18.8 \%$, much higher than below this limit $(5.4 \%)$. This important result of the present study is illustrated in Fig. 3, which shows a histogram of the fraction of Lidetected stars as a function of $J_{0}$. The fraction of Li-detected stars also increases as a function of $\left(J-K_{\mathrm{s}}\right)_{0}$, with a local maximum of $26.3 \%$ between $\left(J-K_{\mathrm{s}}\right)_{0}=0.8$ and 0.9 .

Recently, U07 identified four AGB stars in the PG3 field with Li abundance of $\log \epsilon(\mathrm{Li})=0.8,0.8,1.1$, and 2.0, among a sample of 27 long-period AGB variables. The fraction of upper RGB stars with detectable Li line is similar to that found among AGB stars, accordingly it is possible that the Li-rich AGB stars inherited their Li from the preceding RGB phase. Indeed, the three Li-rich stars identified in the present study could be early AGB stars instead of RGB stars because they are brighter than the RC. Because it is impossible to separate RGB and early AGB 
T. Lebzelter et al.: Lithium in bulge RGB stars

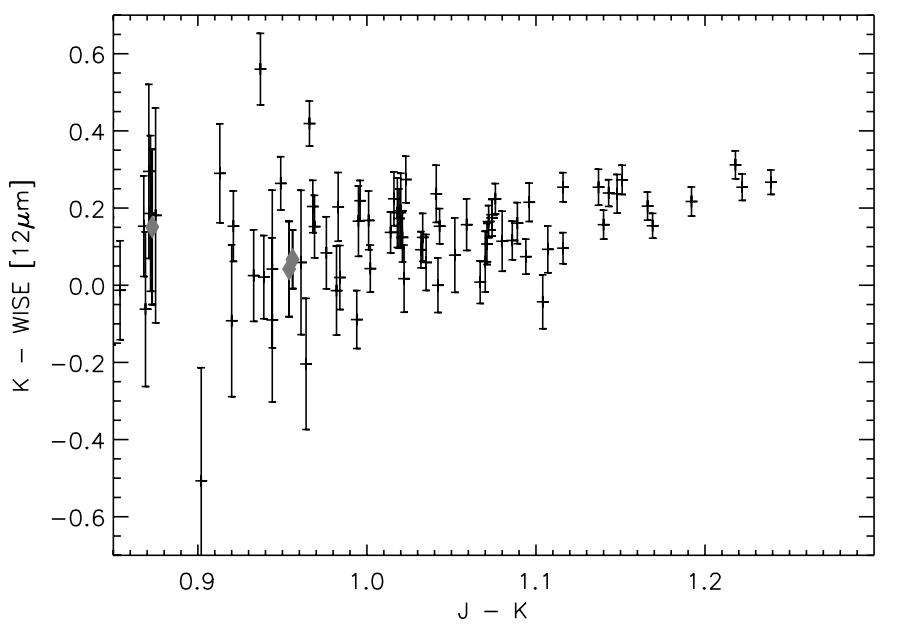

Fig. 4. $K$-WISE[12 $\mu \mathrm{m}]$ vs. $J-K$ colour-colour diagram of our sample stars. The grey diamond symbols represent the three Li-rich stars.

stars by means of our photometric and spectroscopic data alone, asteroseismological methods would be needed to better define their precise evolutionary state.

\subsection{Mass loss from Li-rich stars?}

It has been speculated that the Li enrichment in K-type giants is accompanied by a mass-loss episode (de La Reza et al. 1996, 1997), which has, however, later been questioned (Fekel \& Watson 1998; Jasniewicz et al. 1999). To investigate if there is enhanced dust mass-loss from our target stars, we crossidentified them with sources detected by the WISE space observatory (Wright et al. 2010). Within a search radius of 1'.2 we found counterparts for 333 of our targets in the WISE catalogue ${ }^{4}$. We inspected $J-K$ vs. $K-$ WISE [3.4 $\mu \mathrm{m}$ ], [4.6 $\mu \mathrm{m}$ ], and $[12 \mu \mathrm{m}]$ colour-colour diagrams of these stars. The $22 \mu \mathrm{m}$ WISE band proved to have too low a sensitivity to reliable detect more than just the very brightest of our targets. A version of the $J-K$ vs. $K-$ WISE [12 $\mu \mathrm{m}]$ colour-colour diagram is displayed in Fig. 4. The $x$-axis range was restricted to the reddest part of the sample, because at the bluer (and hence fainter) end of the sample the noise level is very high. The few red outliers in the colour-colour diagrams were identified in the 2MASS images to be visual pairs or triples that are resolved by $2 \mathrm{MASS}$, but are probably unresolved in the WISE observations. The three Lirich stars have very inconspicuous $K$ - WISE colours, strongly suggesting that they do not suffer enhanced dusty mass-loss. For comparison, the Miras investigated by U07, which suffer a total mass-loss rate of $10^{-8} M_{\odot} \mathrm{yr}^{-1}$ or more, have $K-[12 \mu \mathrm{m}]$ colours of 1.17 or redder.

To search also for enhanced gas mass-loss, we inspected the spectra of the Li-rich stars around the $\mathrm{H} \alpha$ line in comparison with otherwise similar Li-poor stars, namely \#051 and \#131. Gas mass-loss would be detectable through blue-shifted asymmetries in the $\mathrm{H} \alpha$ line profile, see e.g. Mészáros et al. (2009) for a sensitive search for gas mass-loss in red giants. The most Li-rich sample star (\#042) has a slightly broader $\mathrm{H} \alpha$ profile than the comparison stars, but no asymmetry. The other two Li-rich stars have a symmetric profile as well. We conclude from this that within the limits of resolution and $\mathrm{S} / \mathrm{N}$ of our spectra, the Li-rich stars do not suffer enhanced loss of either dust nor gas, confirming

\footnotetext{
${ }^{4}$ http://irsa.ipac.caltech.edu/
}

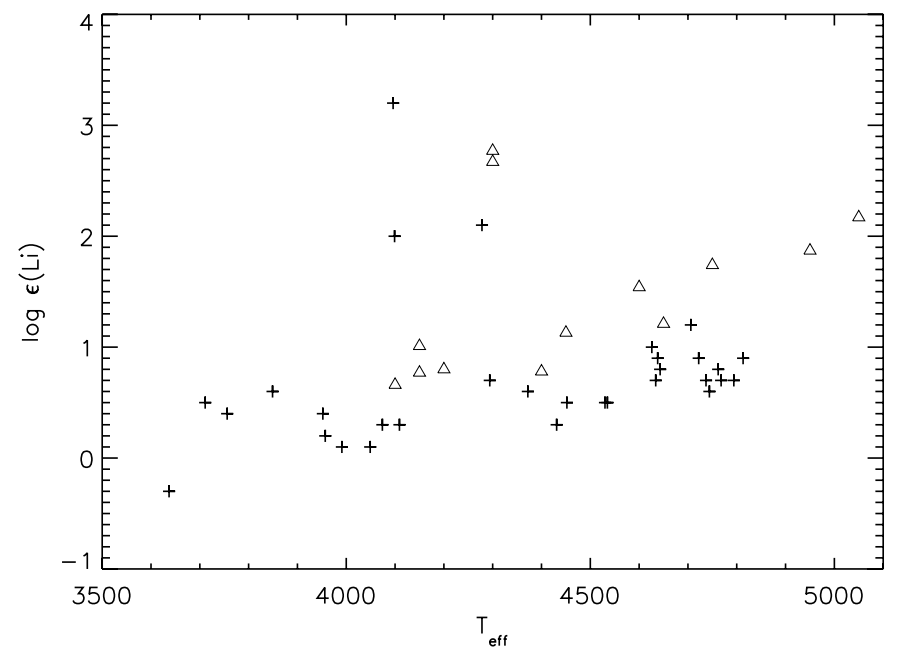

Fig. 5. Lithium abundance as a function of effective temperatures of RGB stars. The data points from our study are plotted as plus symbols, while the open triangles represent the LTE measurements from Gonzalez et al. (2009).

the conclusions of Fekel \& Watson (1998) and Jasniewicz et al. (1999).

\subsection{A trend of Li abundance with temperature}

Recently, a study of Li-rich giants in the GB was presented by Gonzalez et al. (2009). Among a sample of 417 stars, chosen from a narrow brightness range located about 0 . 7 above the expected RC, they found 13 stars with detectable Li line. Gonzalez et al. (2009) concluded that it is unlikely that the Li-rich stars are connected to either the RGB bump or the RC if they are genuine GB stars at a mean distance of $8 \mathrm{kpc}$. Although the authors' sample covers only a narrow luminosity range along the RGB, their results agree with ours. Gonzalez et al. (2009) report a decrease of the Li abundance in the Li-detected stars with decreasing temperature in their sample, which again agrees with our result. A comparison with their LTE results is presented in Fig. 5. Our sample extends the trend found by Gonzalez et al. (2009) to lower temperatures. Just as in Gonzalez et al. (2009), our Li-rich stars also deviate from this trend, and fall in a similar temperature range at $T_{\text {eff }}=\sim 4100-4300 \mathrm{~K}$. One difference to our study is, however, that Gonzalez et al. (2009) find significantly higher Li-abundances at the hot end of their sample ( $T_{\text {eff }} \geq 4600 \mathrm{~K}$ ) than we do. This difference is mostly caused by four stars in the Gonzalez et al. (2009) sample at $T_{\text {eff }} \geq 4600 \mathrm{~K}$ and $\log \epsilon(\mathrm{Li}) \geq 1.5$. Those stars also have a lower surface gravity $(\log g=1.9-2.1)$ than we estimate for our sample stars in this temperature range $(\log g=2.3-2.7)$. Thus, there could be a difference in evolutionary state between these groups of stars, also in terms of $\mathrm{Li}$ abundance, but we are not sure if this fully accounts for the found difference.

Furthermore, Gonzalez et al. (2009) also carefully examined whether the Li-rich stars are special in any other way, but did not find a difference to the Li-normal stars. In a similar attempt, we inspected the spectra of the Li-rich and Li-detected stars in our sample in comparison with Li-poor stars to search for peculiarities, but we did not find any. We only found two sample stars with increased strength in the BaII $649.9 \mathrm{~nm}$ and the LaII $652.9 \mathrm{~nm}$ lines. They probably belong to the so-called barium star class, collecting members of binary systems that owe their enhanced s-element abundances to a mass-transfer episode from 
a more massive AGB companion. These will be discussed in more detail in a forthcoming paper. The Li-rich and Li-detected stars are perfectly normal in their BaII and LaII line strengths, which certifies that their $\mathrm{Li}$ overabundance is not the result of mass transfer of Li-enriched matter from a binary (former AGB) companion. Finally, Gonzalez et al. (2009) also conclude that their Li-rich stars do not suffer enhanced mass-loss, based 2MASS $J H K$ photometry.

The three stars with the highest Li abundance in our sample fall nicely within the sequence of Li-rich thick disk giants identified by Monaco et al. (2011, their Fig. 4). Recently, Kumar et al. (2011) also identified Li-rich giants in the Galactic disk and ascribed them to the red clump, i.e. these are stars after the helium core flash. We found no accumulation of Li-rich stars close to the RCs in our sample. This could be related to a difference in mass between the stars in our study and that of Kumar et al. (2011): While there are mostly low-mass stars with a narrow distribution around $1 M_{\odot}$ in our sample, the disk sample of Kumar et al. (2011) likely also contains stars of higher mass, whose Li abundance may evolve differently. The thick disk sample of Monaco et al. (2011) likely contains mostly stars of low mass as well, although the authors identify one star that could have a mass of up to $4 M_{\odot}$, albeit with large uncertainty. The agreement between our results and those of Monaco et al. (2011) is probably related to a similar mass spectrum of the samples.

\section{Conclusions}

There are now several studies that show that at low to intermediate masses Li-rich stars can be found all along the RGB, not necessarily connected to a particular phase of the giant branch evolution (Gonzalez et al. 2009; Monaco et al. 2011; Alcalá et al. 2011; Ruchti et al. 2011). This means that either the Li enrichment process is stochastic in nature and can happen at any time on the $\mathrm{RGB}$, or that $\mathrm{Li}$ enrichment starts at some point on the lower RGB and may take a long time to make Li-rich $\mathrm{K}$ giants. The activation of mixing events of variable duration and transport rates below the convective envelope is conceivable for the Li enrichment (Palmerini et al. 2011). The lack of a well-defined Li-rich phase on the RGB, as now shown by several recent studies, contradicts predictions by $\mathrm{CB} 00$, who propose that a $\mathrm{Li}$-rich phase should occur at the bump of the RGB in low-mass stars, such as those that we have in our sample. The existence of such a Li-rich phase has to be questioned.

Acknowledgements. T.L. acknowledges support from the Austrian Science Fund (FWF) under projects P 21988-N16 and P 23737-N16, and SU under project P 22911-N16. B.A. thanks for the support from contract ASI-INAF I/009/10/0. This publication makes use of data products from the Two Micron All Sky Survey, which is a joint project of the University of Massachusetts and the Infrared Processing and Analysis Center/California Institute of Technology, funded by the National Aeronautics and Space Administration and the National Science Foundation. This publication makes use of data products from the Widefield Infrared Survey Explorer, which is a joint project of the University of California, Los Angeles, and the Jet Propulsion Laboratory/California Institute of Technology, funded by the National Aeronautics and Space Administration.

\section{Appendix A: The $\left(J-K_{S}\right)-T_{\text {eff }}$ calibration from COMARCS models}

In Table A.1 and Fig. A.1 we present an effective temperature calibration for the 2MASS $J-K_{\mathrm{S}}$ colour based on hydrostatic COMARCS atmospheres and the COMA spectral synthesis package (see Aringer et al. 2009). The same input opacities were used for the construction of the models and the subsequent

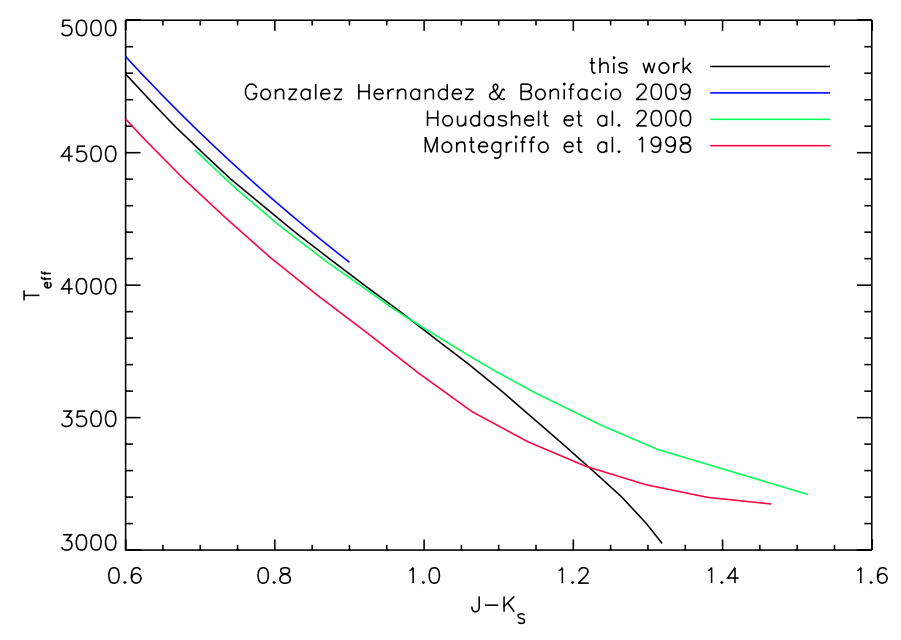

Fig. A.1. Comparison between different $\left(J-K_{\mathrm{S}}\right)-T_{\text {eff }}$ calibrations as identified in the legend.

Table A.1. $\left(J-K_{\mathrm{S}}\right)-T_{\text {eff }}$ calibration from COMARCS models.

\begin{tabular}{lccccc}
\hline \hline$T_{\text {eff }}$ & $J-K_{\mathrm{S}}$ & $\log g$ & $\mathrm{BC}(\mathrm{J})$ & $\mathrm{BC}(\mathrm{H})$ & $\mathrm{BC}(\mathrm{K})$ \\
\hline 5800 & 0.343 & 4.24 & 1.108 & 1.404 & 1.451 \\
5700 & 0.363 & 4.12 & 1.141 & 1.453 & 1.504 \\
5600 & 0.384 & 4.05 & 1.174 & 1.502 & 1.558 \\
5500 & 0.407 & 4.01 & 1.205 & 1.552 & 1.612 \\
5400 & 0.430 & 3.98 & 1.237 & 1.603 & 1.667 \\
5300 & 0.454 & 3.95 & 1.268 & 1.653 & 1.722 \\
5200 & 0.481 & 3.92 & 1.298 & 1.705 & 1.779 \\
5100 & 0.509 & 3.88 & 1.327 & 1.757 & 1.836 \\
5000 & 0.538 & 3.82 & 1.356 & 1.810 & 1.894 \\
4900 & 0.569 & 3.70 & 1.385 & 1.864 & 1.954 \\
4800 & 0.599 & 3.35 & 1.417 & 1.919 & 2.016 \\
4700 & 0.632 & 3.03 & 1.447 & 1.973 & 2.079 \\
4600 & 0.666 & 2.77 & 1.477 & 2.028 & 2.143 \\
4500 & 0.703 & 2.52 & 1.505 & 2.083 & 2.208 \\
4400 & 0.741 & 2.31 & 1.532 & 2.138 & 2.273 \\
4300 & 0.784 & 2.11 & 1.557 & 2.193 & 2.341 \\
4200 & 0.827 & 1.91 & 1.582 & 2.247 & 2.409 \\
4100 & 0.873 & 1.72 & 1.606 & 2.302 & 2.479 \\
4000 & 0.920 & 1.54 & 1.630 & 2.357 & 2.550 \\
3900 & 0.968 & 1.35 & 1.656 & 2.412 & 2.624 \\
3800 & 1.014 & 1.17 & 1.685 & 2.469 & 2.699 \\
3701 & 1.060 & 1.00 & 1.716 & 2.526 & 2.776 \\
3600 & 1.104 & 0.82 & 1.752 & 2.586 & 2.856 \\
3500 & 1.145 & 0.67 & 1.792 & 2.646 & 2.937 \\
3400 & 1.186 & 0.49 & 1.835 & 2.705 & 3.021 \\
3300 & 1.226 & 0.33 & 1.879 & 2.761 & 3.105 \\
3200 & 1.265 & 0.17 & 1.923 & 2.817 & 3.188 \\
3100 & 1.298 & 0.03 & 1.966 & 2.873 & 3.264 \\
3025 & 1.319 & -0.11 & 1.998 & 2.912 & 3.317 \\
\hline & & & & &
\end{tabular}

radiative transfer calculations. A list including most of the incorporated molecular species and data can be found in Marigo $\&$ Aringer (2009). The calculations are consistent with the spectral synthesis applied in this work to obtain metallicities and Li abundances.

For the presented models we assumed a microturbulent velocity of $\xi=2.5 \mathrm{~km} \mathrm{~s}^{-1}$, solar abundances from Grevesse \& Sauval (1998), with revisions from Caffau et al. (2008, 2009) and surface gravities based on a typical solar mass and abundance evolutionary sequence (Marigo et al. 2008). Compared to the effective temperature, the influence of metallicity and surface gravity on the predicted $J-K_{\mathrm{s}}$ colours is fairly small. We expect 
a maximum uncertainty of $\pm 50 \mathrm{~K}$ for our programme stars owing to the variation of these quantities.

A comparison between different $\left(J-K_{\mathrm{S}}\right)-T_{\text {eff }}$ calibrations is shown in Fig. A.1. Our calibration (black graph) is compared to those of González Hernández \& Bonifacio (2009, blue graph), Houdashelt et al. (2000, green graph), and of Montegriffo et al. (1998, red graph). The relations of Houdashelt et al. (2000) and Montegriffo et al. (1998) were transformed from the Bessel \& Brett (Bessel \& Brett 1988) and ESO (van der Bliek et al. 1996) systems, respectively, to the 2 MASS system using the relations of Carpenter (2001). Solar metallicity $([\mathrm{Fe} / \mathrm{H}]=0.0)$ was assumed for the relation of González Hernández \& Bonifacio (2009), but the dependence on metallicity is weak. This last relation is only valid up to $J-K_{\mathrm{S}}=0.9$, but an extrapolation to redder values agrees well with the other relations. The relation of Montegriffo et al. (1998) results in lower temperatures than the others by about $200 \mathrm{~K}$ in a broad range of $J-K_{\mathrm{S}}$ values, whereas the difference between the other three relations, in the region of overlap, is small. Our relation agrees particularly well with that of Houdashelt et al. (2000) up to $J-K_{\mathrm{S}} \sim 1.1$, but it subsequently diverges.

\section{References}

Alcalá, J. M., Biazzo, K., Covino, E., Frasca, A., \& Bedin, L. R. 2011, A\&A, 531, L12

Aringer, B. 2000, Ph.D. Thesis, University of Vienna, Austria

Aringer, B., Girardi, L., Nowotny, W., et al. 2009, A\&A, 503, 913

Bessell, M. S., \& Brett, J. M. 1988, PASP, 100, 1134

Brown, J. A., Sneden, C., Lambert, D. L., et al. 1989, ApJS, 71, 293

Caffau, E., Ludwig, H.-G., Steffen, M., et al. 2008, A\&A, 488, 10311

Caffau, E., Maiorca, E., Bonifacio, P., et al. 2009, A\&A, 498, 877

Cameron, A. G. W., \& Fowler, W. A. 1971, ApJ, 164, 111

Carpenter, J. M. 2001, AJ, 121, 2851

Charbonnel, C., \& Balachandran, S. C. 2000, A\&A, 359, 563; CB00 de La Reza, R., Drake, N. A., \& da Silva, L. 1996, ApJ, 456, L115

de La Reza, R., Drake, N. A., da Silva, L., et al. 1997, ApJ, 482, L77

De Propris, R., Rich, R. M., Kunder, A., et al. 2011, ApJ, 732, L36

Fekel, F. C., \& Watson, L. C. 1998, AJ, 116, 2466

Girard, T. M., van Altena, W. F., Zacharias, N., et al. 2011, AJ, 142, 15

Girardi, L., Bressan, A., Bertelli, G., \& Chiosi, C. 2000, A\&AS, 141, 371
Girardi, L., Groenewegen, M. A. T., Hatziminaoglou, E., \& da Costa, L. 2005, A\&A, 436, 895

Glass, I. S., \& Schultheis, M. 2003, MNRAS, 345, 39

Gonzalez, O. A., Zoccali, M., Monaco, L., et al. 2009, A\&A, 508, 289

González Hernández, J. I., \& Bonifacio, P. 2009, A\&A, 497, 497

Grevesse, N., \& Sauval, A. J. 1998, SSRv, 85, 161

Hinkle, K., Wallace, L., Valenti, J., \& Harmer, D. 2000, Visible and Near Infrared Atlas of the Arcturus Spectrum 3727-9300 $\AA$ (San Francisco: ASP)

Houdashelt, M. L., Bell, R. A., Sweigart, A. V., \& Wing, R. F. 2000, AJ, 119, 1424

Jasniewicz, G., Parthasarathy, M., de Laverny, P., \& Thévenin, F. 1999, A\&A, 342,831

Kumar, Y. B., Reddy, B. E., \& Lambert, D. L. 2011, ApJ, 730, L12

Kupka, F., Piskunov, N., Ryabchikova, T. A., Stempels, H. C., \& Weiss, W. W. 1999, A\&AS, 138, 119

Lambert, D. L., Dominy, J. F., \& Sivertsen, S. 1980, ApJ, 235, 114

Lind, K., Asplund, M., \& Barklem, P. S. 2009a, A\&A, 503, 541

Lind, K., Primas, F., Charbonnel, C., Grundahl, F., \& Asplund, M. 2009b, A\&A, 503,545

Mallik, S. V. 1999, A\&A, 352, 495

Marigo, P., \& Aringer, B. 2009, A\&A, 508, 1539

Marigo, P., Girardi, L., \& Bressan, A. 2008, A\&A, 482, 883

McWilliam, A., \& Zoccali, M. 2010, ApJ, 724, 1491

Mészáros, Sz., Dupree, A. K., \& Szalai, T. 2009, AJ, 137, 4282

Michaud, G. 1986, ApJ, 302, 650

Mishenina, T. V., Bienaymé, O., Gorbaneva, T. I., et al. 2006, A\&A, 456, 1109

Monaco, L., Villanova, S., Moni Bidin, C., et al. 2011, A\&A, 529, A90

Montegriffo, P., Ferraro, F. R., Origlia, L., \& Fusi Pecci, F. 1998, MNRAS, 297, 872

Nataf, D. M., Udalski, A., Gould, A., Fouqué, P., \& Stanek, K. Z. 2010, ApJ, 721, L28

Palmerini, S., Cristallo, S., Busso, M., et al. 2011, ApJ, 741, 26

Pasquini, L., Randich, S., \& Pallavicini, R. 2001, A\&A, 374, 1017

Pasquini, L., Randich, S., Zoccali, M., et al. 2004, A\&A, 424, 951

Robin, A. C., Reylé, C., Derrière, S., et al. 2003, A\&A, 409, 523

Ruchti, G. R., Fulbright, J. P., Wyse, R. F. G., et al. 2011, ApJ, 743, 107

Schlegel, D. J., Finkbeiner, D. P., \& Davis, M. 1998, ApJ, 500, 525

Ryde, N., Gustafsson, B., Edvardsson, B., et al. 2010, A\&A, 509, A20

Schultheis, M., Ng, Y. K., Hron, J., \& Kerschbaum, F. 1998, A\&A, 338, 581

Skrutskie, M. F., Cutri, R. M., Stiening, S., et al. 2006, AJ, 131, 1163

Uttenthaler, S., Lebzelter, T., Palmerini, S., et al. 2007b, A\&A, 471, L41; U07

van der Bliek, N. S., Manfroid, J., \& Bouchet, P. 1996, A\&AS, 119, 547

Vanhollebeke, E., Groenewegen, M. A. T., \& Girardi, L. 2009, A\&A, 498, 95

Wright, E. L., Eisenhardt, P. R. M., Mainzer, A. K., et al. 2010, AJ, 140, 1868

Zoccali, M., Hill, V., Lecureur, A., et al. 2008, A\&A, 486, 177 